\title{
Analysis of Electrical Characteristics of Inter-wire Arc in Cross-Coupling Arc
}

\author{
Zhenyang Lu, Shanwen Dong, Fan Jiang ${ }^{*}$ and Cheng Li
}

\begin{abstract}
As a new composite welding heat source introduced in recent years, the cross-coupling arc uses a non-consumable electrode arc (plasma arc) and a consumable electrode arc (inter-wire arc) in cross-coupling, in which the plasma arc is the main arc and the inter-wire arc is the vice arc, to realize the separate control of the heat input to the workpiece, arc force, and droplet transfer. To reveal the electrical characteristics of the inter-wire arc under the action of the plasma arc, in this study, rotating probes are used to sweep across the plasma arc. When the probes rotate into the plasma arc, a circuit is formed, and the electrical characteristics of the inter-wire arc are indirectly analyzed according to the circuit: the probe centering current and input voltage are used as the physical quantities. The results show that at a certain wire feed rate, the inter-wire arc current increases with increasing input voltage. When the input voltage is low, the wire feed rate has no obvious effect on the inter-wire arc current. At a higher input voltage, where the wire feed rate is high, the inter-wire arc current decreases. With the increase in the plasma arc current, the inter-wire arc current first increases quickly and then increases slowly, and simultaneously, the striking arc time becomes longer. With the increase in the interval between the nozzle and the workpiece, the inter-wire arc current increases, but when the arc length increases to a certain limit, the slope of the welding current clearly declines.
\end{abstract}

Keywords: Inter-wire arc, Circuit, Input voltage, Inter-wire arc current

\section{Introduction}

As an important means of metal material processing, arc welding has many advantages, such as low cost, ease of operation and high automation [1-5]. However, traditional arc welding technology fails to satisfy the demand for the rapid development of industrial welding. Domestic and foreign scholars proposed a new type of multielectrode arc welding technology. Zhang et al. [6-9] at the University of Kentucky proposed double-electrode gas metal arc welding (DE-GMAW), in which a bypass electrode is used to reduce the heat input to workpiece and to ensure the deposition rate. In 2012, Zhang et al. [10-14] proposed bypass arcing-wire gas metal arc welding (arcing-wire GTAW), which uses a non-consumable electrode arc of a main road and a consumable electrode arc of a bypass in coupling. To realize the decoupled

\footnotetext{
*Correspondence: jiangfan@bjut.edu.cn

College of Mechanical Engineering and Applied Electronics Technology, Ministry of Education Engineering Research Center of Advanced Manufacturing Technology for Automotive Components, Beijing University of Technology, Beijing 100124, China
}

control of the weld depth and deposition, the main arc controls the weld penetration, and the bypass arc controls the wire deposition. Chen et al. $[15,16]$ at Beijing University of Technology proposed cross-coupling arc welding based on DE-GMAW and arcing-wire GTAW. In Figure 1, a cross-coupling arc combines a non-consumable electrode arc and a consumable electrode arc. The non-consumable electrode arc, as the main arc, is formed between the workpiece and the electrode and mainly controls the heat and force input to the workpiece. The consumable electrode arc, as the inter-wire arc, burns between the two wires and mainly controls the wire deposition and the heat input to the workpiece. The molten pool is only used as an electrode of a cross-coupling arc. It greatly reduces the thermal input and has no electrical connection with the arc welding circuit between wires. A cross-coupling arc realizes decoupled control of the heat transfer, force transfer, and mass transfer in the welding process. Cross-coupling arc welding technology breaks the traditional concept of "welding arc is the strong and lasting discharge phenomenon between 


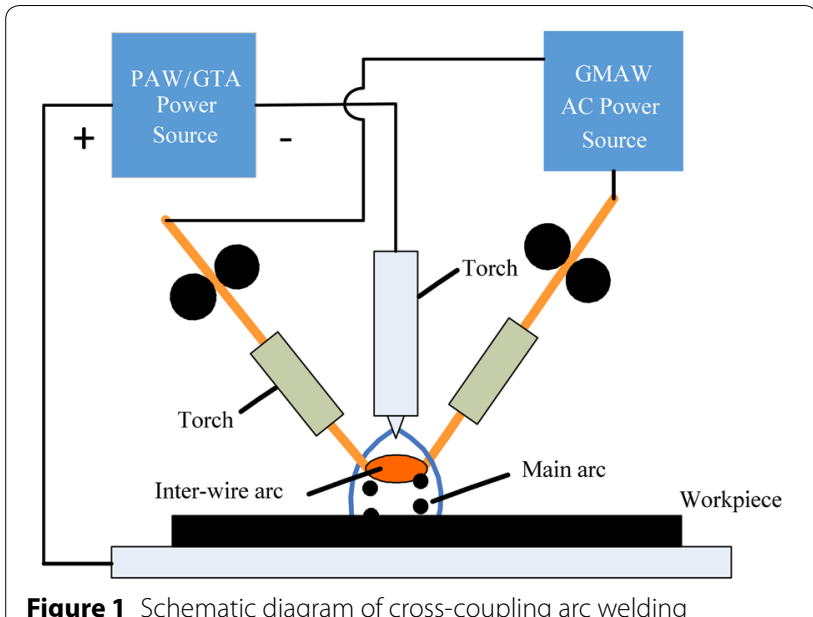

Figure 1 Schematic diagram of cross-coupling arc welding the two electrodes". The multiple electrodes discharge simultaneously in the arc space, and the molten pool is only a function object of multi-electrode arcs. The multiarc coupling carrier is the arc itself [17-20]. The crosscoupling arc mode fundamentally relieves the inherent constraints of the traditional arc in heat transfer, force transfer and mass transfer, and provides a new technical method for achieving high-efficiency, high-speed and high-quality arc welding. The research on cross-coupling arc welding mainly focuses on the welding process and the coordination control of the welding equipment. However, the research on the electrical characteristics of the cross-coupling arc is insufficient, and these characteristics determine the stability of the arc-power source system, and affect the final welding quality. Therefore, further analysis of the electrical characteristics of a crosscoupling arc could optimize and improve the cross-coupling arc welding technology.

A series of methods have been employed to study the electrical characteristics of the arc [21-24]. Li et al. [2527] at Xi'an Shiyou University used differential analysis of an electrostatic probe with low disturbance to diagnose the current carrying area of a tungsten inert gas (TIG) arc and measured the saturated ionic current to solve different welding currents. Chen et al. [28, 29] at Beijing University of Technology invented a device and a method to detect the arc characteristics. The curve of the arc characteristic was obtained by collecting and processing the voltage signals between the two probes. Lu et al. $[30,31]$ at Shenyang University of Technology employed probes to test the radial distribution of the arc current density under the action of high-frequency magnetic pulses. Lv et al. [32, 33] at Tianjin University described the electrical characteristics of the arc by simulating a TIG arc using MATLAB software. The most significant feature of the cross-coupling arc is that the welding arc is in a coupling state, which differs from a traditional composite arc. A traditional composite arc is composed of multiple arcs side-by-side at a certain angle, as with the twin-wire TANDEM welding arc and tri-wire welding arc. The composite electrical properties basically maintain the electrical characteristics of each arc, but a cross-coupling arc comprises multiple arcs in cross-coupling, combining the transverse electrical characteristic and the longitudinal electrical characteristic of each arc. This inevitably changes the electrical characteristics of the coupling arc. The aforementioned methods can only measure the electrical signals of the cross-coupling arc; they cannot directly and effectively elucidate the influence of the plasma arc on the electrical characteristics of the inter-wire arc or the effect of the inter-wire arc on the electrical characteristics of the plasma arc within the cross-coupling arc.

In this study, the inter-wire arc of a cross-coupling arc was the research object, and an arc test system based on rotating probes was built. Instead of an inter-wire arc, a small signal model was used. This paper reports the current-voltage characteristic of the inter-wire arc within the plasma arc, as well as the effects of the plasma arc and the spatial position of the inter-wire arc on the current-voltage characteristics. This lays the foundation for further study of the arc characteristics and the arcing mechanism of cross-coupling arcs.

\section{Experimental Procedures}

\subsection{Experimental Equipment and Principles}

Because an inter-wire arc is a double consumable electrode arc, there is a real-time variation of the arc length. When studying the action of the plasma arc on the interwire arc and the relationship between the welding parameters of the inter-wire arc under the plasma arc, the interference factor of arc length variation will be added. This makes the analysis of the test results more difficult. Therefore, the non-consumable electrode arc was considered. Additionally, to avoid the test background of the plasma arc being changed by the inter-wire arc parameters, it was possible to reduce the influence of the plasma arc background through small signal models. By integrating these two factors, the inter-wire arc welding circuit was replaced by a probe circuit. The movement of the probe and the welding wire into the plasma arc was neglected. The rotating speed of the probe replaced the wire feeding speed of the inter-wire arc, and the center current of the probe replaced the arc current between wires. The plasma arc was used as a non-consumable electrode arc in the experiment, which did not produce 
droplet transition and had little effect on the probe measurement.

A connection between the probe circuit and inter-wire arc circuit was established by rotating the probes through the plasma arc. We used the rotation mode of the probes to avoid them being burnt out by the high center temperature of the plasma arc. Because the inter-wire arc was a consumable electrode arc, droplet transfer existed in the welding process, and the wires conducted droplet transfer stably in the relatively fixed position of the plasma arc. By setting the position of the probe end and the position of the wire end to coincide, the wire feed rate was replaced with the probe rotation rate.

In the experiment, two probes, each having a diameter of $1.0 \mathrm{~mm}$ were used as electrodes. The probes were fixed on the axis of the stepper motor and swept through the plasma arc owing to motor rotation. Figure 2 shows the probes were rotating into the arc. The surface of the probes was coated with a high-temperature insulating coating (except the end). On one hand, because of the high temperature of the plasma arc column, the probes were relatively thin and easy to burn; on the other hand, the current was guaranteed to pass through only a section of the probe end.

As shown in Figure 3, the output of the VPPA-400A plasma arc welding power source formed a stable plasma arc between the welding torch and the water-cooled copper block, and the plasma arc was controlled by the external characteristic of the constant current. The probe detection circuit consisted of an adjustable voltage source, a resistance and two probes. On one hand, the resistance played a role in limiting the current, preventing the current in the probes from being too high and burning the voltage source. On the other hand, it acted as a display contrast, whereby the equivalent voltage between the probes and the circuit current could be calculated from the resistance.

The rotating probes mainly penetrated the outside of the plasma arc; thus, the tips of the probes were exposed to the arc column. Under the electric-field action at the end of the probes, the electrons escaped from and

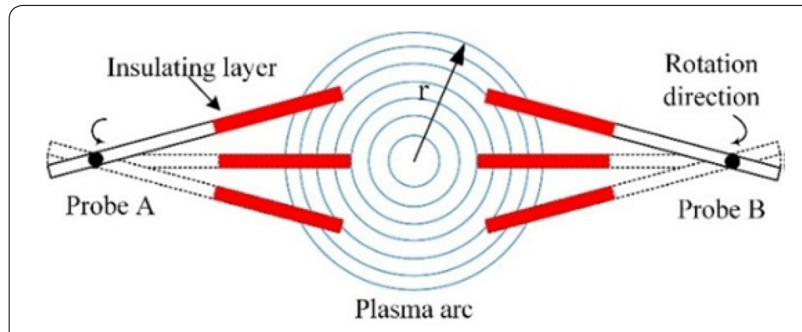

Figure 2 Diagram of the probes rotating into the plasma arc

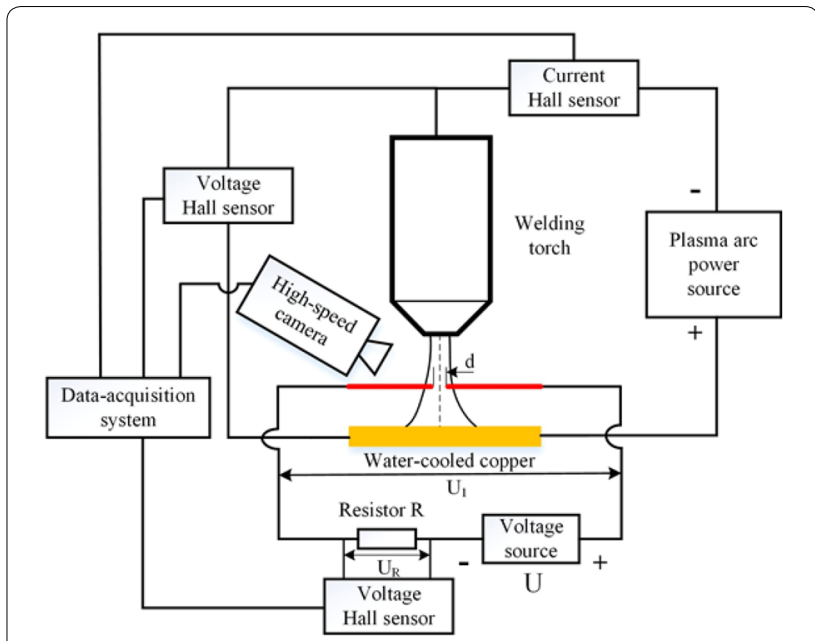

Figure 3 Schematic diagram of the experimental principle

entered the ends of the probes, forming a circuit current. When the probes rotated into the arc column, a circuit current was formed between the probes, and the voltage waveform between the probes was shown in Figure 4.

Figure 5 shows a diagram of the changes of the probe voltage and the resistance voltage with respect to time in a rotation period. The two curves change with the same trend; that is, when the probes rotate into the plasma arc, the probe voltage and the resistance voltage follow Kirchhoff's voltage law at any given time. When the resistance voltage is zero, the probe circuit is open, and the probes do not enter the plasma arc. When the resistance voltage starts increasing from zero to the extreme point and then decreases to zero, the probe circuit is closed with current passing through the probes, and there is a voltage between the probes. The trend of the change in the probe voltage is similar to the sinusoidal function. When the minimum probe voltage is reached, the end face of the probe is parallel to that of the other probe, and the interval between the two probes is minimized.

When the probes rotate into the plasma arc, a circuit is formed between them. The resistance voltage and the circuit current were calculated according to Kirchhoff's law. The resistance voltage was calculated using Eq. (1), and the circuit current was calculated using Eq. (2). Thus, the electrical characteristics of the inter-wire arc within the cross-coupling arc were studied.

$$
\begin{aligned}
& U_{\mathrm{R}}=U_{\mathrm{i}}-U_{1}, \\
& I_{1}=\frac{U_{\mathrm{R}}}{R},
\end{aligned}
$$

where $U_{\mathrm{i}}$ is the input voltage, $U_{\mathrm{R}}$ is the resistance voltage, and $R$ is the resistance value. 


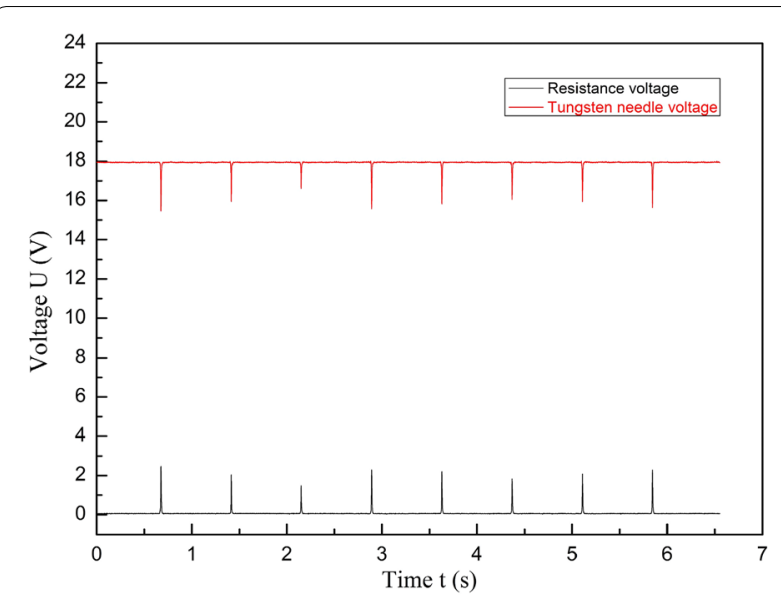

Figure 4 Waveform diagram of the probe voltage and the resistance voltage

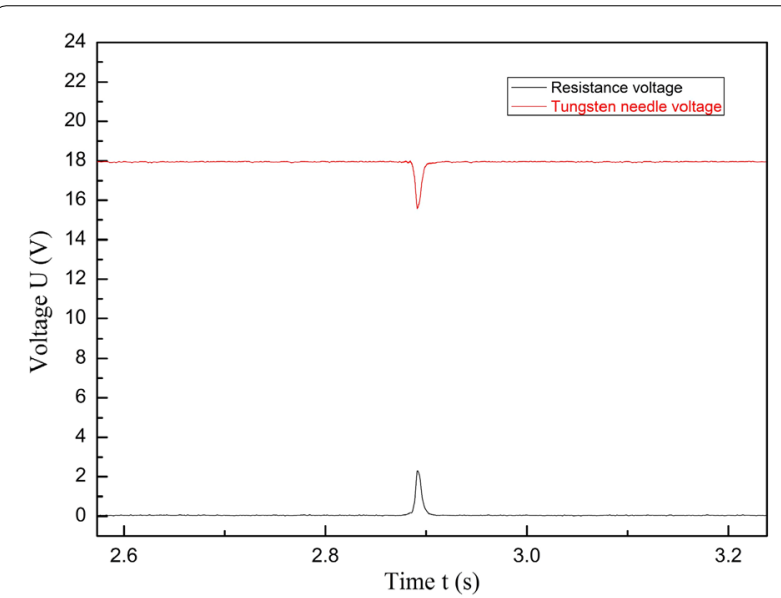

Figure 5 Enlarged graph of the probe voltage and the voltage waveforms of the resistance

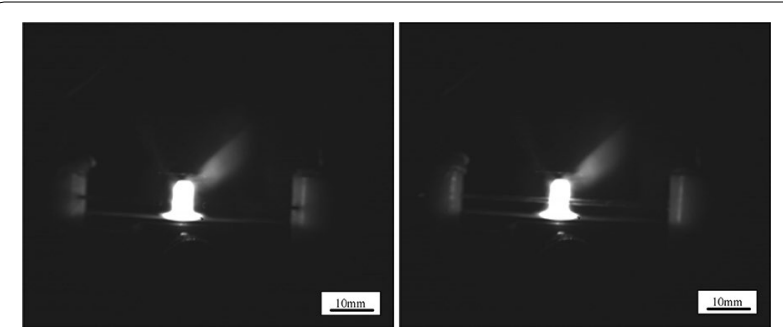

Figure 6 State of the probes before they rotate into the plasma arc and state of the probes within the plasma arc

As shown in Figure 6, the processes occurring when the probes rotated into the plasma arc were captured and recorded in real time using a high-speed camera.
Before the probes rotated into the arc, the plasma arc was in a bundle, and when the probes rotated into the arc, the outer contour of the plasma arc remained basically unchanged. In the cross-coupling arc welding process, the inter-wire arc burned in the plasma arc. The inter-wire arc did not affect the high binding state of the plasma arc, and the plasma arc force was still obvious.

\subsection{Schemes of Experiments}

As shown in Table 1, Experiment \#1 was designed to study the influence of the wire feed rate on the electrical characteristics of the inter-wire arc and the self-regulation of the inter-wire arc within the plasma arc. By changing the probe speed and input voltage of the probe circuit when the probes rotated into the plasma arc, the probe voltage was measured, and the probe centering current was calculated. Then, the relationship between the probe centering current and the input voltage of the probe circuit was determined at different probe speeds. Experiment \#2 was designed to analyze the effect of the plasma arc on the inter-wire arc. The influence of the plasma arc current on the inter-wire arc current and striking arc time was discussed in detail. At a certain probe speed, when the plasma arc current was changed, the trends of the changes of the probe centering current and of the centering time were studied. Finally, Experiment \#3 was designed to study the influence of the interwire arc location on the inter-wire arc current, and the change rule of the probe centering current was obtained by changing the interval between the torch nozzle and the workpiece.

In the experiments, the plasma gas flow rate was $2 \mathrm{~L} /$ min. The shielding gas flow rate was $15 \mathrm{~L} / \mathrm{min}$. The resistance $R$ was $100 \Omega$. The distance from the probe to the water-cooled copper block was $4 \mathrm{~mm}$, and the interval between the probes was $4 \mathrm{~mm}$. A data-acquisition system recorded the welding current, the probe voltage, and the resistance voltage in real time. Its sampling rate was as high as $10 \mathrm{k} / \mathrm{s}$. A high-speed camera recorded the arc shape at a frame rate of $3000 \mathrm{f} / \mathrm{s}$.

\section{Results and Discussion}

\subsection{Electrical Characteristics of Inter-wire Arc Under Plasma Arc}

Figure 7 shows a trend diagram of the probe centering current with respect to the input voltage of the probe circuit at different probe speeds. The curve in the figure was obtained in Experiment \#1. As shown in the diagram, at the same probe speed, with the increase in the input voltage of the probe circuit, the circuit current increases, but when the input voltage is approximately $24 \mathrm{~V}$, the current changes obviously from a slow rise to a rapid rise. At the same input voltage of the probe circuit, the change 
Table 1 Experiment parameters

\begin{tabular}{lllll}
\hline No. & $\begin{array}{l}\text { Speed } \\
\boldsymbol{v}(\mathbf{r a d} / \mathbf{s})\end{array}$ & $\begin{array}{l}\text { Welding current } \\
\boldsymbol{I}(\mathbf{A})\end{array}$ & $\begin{array}{l}\text { Interval } \\
\boldsymbol{h}(\mathbf{m m})\end{array}$ & $\begin{array}{l}\text { Input voltage } \\
\boldsymbol{U}_{\mathbf{i}}(\mathbf{V})\end{array}$ \\
\hline$\# 1$ & 3.55 & 100 & 10 & $12.3,18.0,23.7,29.6$, \\
& 7.25 & & & 35.3 \\
& 9.34 & & \\
& 11.52 & & \\
& 20.16 & & 23.7 \\
& 21.20 & & \\
$\# 2$ & 7.25 & $100,130,145,160$, & 10 \\
& \multicolumn{4}{c}{$190,200,220}$, \\
$\# 3$ & 7.25 & 100 & $5,6,7,8,10$ & 23.7 \\
\hline
\end{tabular}

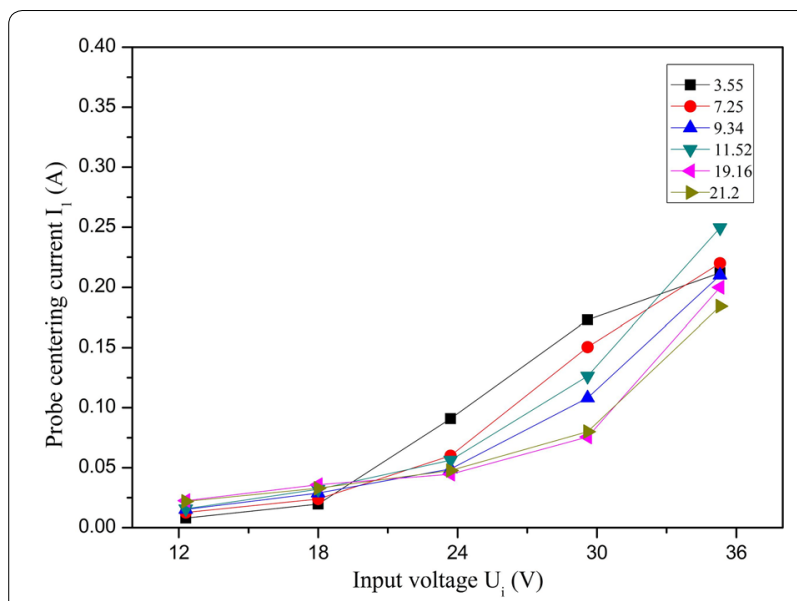

Figure 7 Relationship between the probe centering current and the input voltage at different probe speeds

trend of the probe centering current varies because the probe speed varies. When the input voltage $U_{\mathrm{i}}$ is $<20 \mathrm{~V}$, a higher probe speed yields a larger centering current, but the centering current remains basically unchanged because of the low input voltage. When the input voltage $U_{\mathrm{i}}$ is $>20 \mathrm{~V}$, the change in the probe centering current is distinct. A lower probe speed yields a larger probe centering current.

When the probe circuit has an input voltage and the probes start to rotate into the arc, the electrons stored beforehand in the probe tips are heated to an excited state. Under the force of the electric field, electrons escape from the probe surface and move directionally, forming the circuit current. The electron source has two parts: the electron flow in the plasma arc and the electrons provided by the probe circuit. In the plasma arc, the direction of electron motion is from the tungsten electrode to the water-cooled copper block. In the probe circuit, the direction of electron motion is from the cathode of the power source to the anode. The electric-field action between the plasma arc and the probe circuit leads to deflection of the electron movement to the opposite side, increasing the resistance of the electron movement. As shown in Figure 7, the input voltage of the probe circuit is higher. On one hand, according to the capacitor formula $C=Q / U$, a larger amount of electrons stored at the end of the probe yields a larger amount of electrons excited by the heat of the arc. On the other hand, the electric field between the probes becomes stronger, and the time for electrons to escape from and enter the probe tips is reduced. That is, the number of electrons escaping from the probe surface and entering the probe end per unit time increases, along with the circuit current.

Further analysis of the input voltage higher than $20 \mathrm{~V}$ was performed because the arc voltage cannot be too low in the cross-coupling arc welding process, owing to the existence of the polar voltage. At a certain wire feed rate, a higher input voltage of the inter-wire arc yields a greater increase in the welding circuit current. Moreover, the rising characteristic of the inter-wire arc is similar to the rising section of the static characteristic curve of a traditional TIG arc, as shown in Figure 8. When the input voltage of the inter-wire arc increases, the electric field between the inter-wire arc becomes stronger; thus, the number of electrons entering the wire end per unit time increases, the welding circuit current increases, and the lateral resistance of the plasma arc column decreases. When the inter-wire arc circuit is controlled by a constant voltage source, a higher wire feed rate yields less time for preheating the end of the wire. Moreover, with the increase in the wire feed rate, the number of electrons escaping from the cathode area decreases, along with the inter-wire arc current.

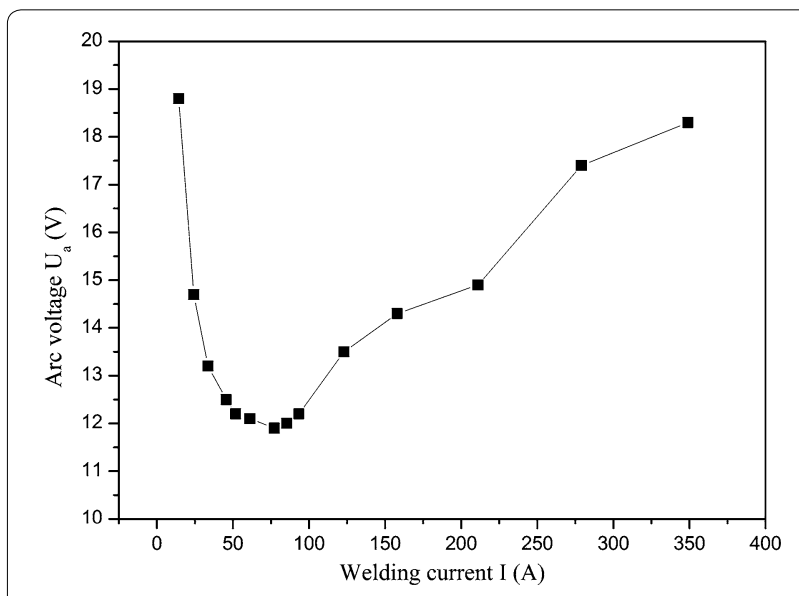

Figure 8 Static characteristic of a traditional TIG arc 
According to the results of Experiment \#1, as shown in Figure 7, the probe centering current increases with the increase in the probe input voltage under the plasma arc background and with the increase in the probe rotation speed. At the same input voltage, a higher probe speed yields a smaller probe current. In the process of cross-coupling arc welding, the inter-wire arc acts as the consumable electrode arc, and droplets are forced to transfer to the molten pool under the thermal action of the plasma arc. Thus, droplet transfer is completed in the plasma arc. When the plasma arc parameters are fixed, the inter-wire arc current increases with the arc input voltage. Because of the increase in the input voltage and current, the heat production in the cathode and anode regions of the inter-wire arc increase, and the stable droplet transition point at the end of the inter-wire arc wire deviates from the central line of the plasma arc. That is, the distance between the two welding wires increases; accordingly, the heat of the inter-wire arc is reduced by the plasma arc. A higher wire feeding speed yields a smaller inter-wire arc current and less arc heating power, given the same input voltage. To obtain more heat, the stable droplet transition point must be closer to the plasma arc center line; that is, the distance between the two wires must be reduced.

\subsection{Influence of Plasma Arc on Inter-wire Arc Current and Striking Arc Time of Inter-wire Arc}

Figure 9 shows a trend diagram of the circuit current with the increase in the plasma arc current. The curve in the figure was obtained from Experiment \#2. With the increase in the plasma arc current, the probe centering current increases. The difference in the curve slope of the welding current before and after $140 \mathrm{~A}$ is obvious, but the curve remains linear. When the welding current is $<140 \mathrm{~A}$, the centering current increases faster with the increase in the welding current, and when the welding current is $>140 \mathrm{~A}$, the probe centering current increases gently.

With the increase in the plasma arc current, the arc energy increases, the electrons have more excitation energy at the tip of the probes, and it becomes easier for them to escape from the probe surface. At the same time, the number of electrons in the plasma arc increases. Finally, under the force of the electric field, the number of electrons entering the end of the probes per unit time increases, and the circuit current of the probe increases accordingly. When the welding current is $>140 \mathrm{~A}$, with the increase in the welding current, the electron absorption energy and the number of electrons in the plasma arc volume increase. However, owing to the limitation of the probe tip area and the resistance of the circuit, the number of electrons entering the probe tips tends to be saturated per unit time, and the circuit current increases slowly. In the cross-coupling arc welding process, the inter-wire arc is controlled by the constant voltage characteristic. Under the premise of a certain wire feed rate, the inter-wire arc current increases with the plasma arc current; furthermore, the heat power of the arc column increases with the plasma arc current. In this case, the wire end is fully preheated, and the ionization degree in the volume between the plasma arc and the inter-wire arc increases. Thus, the number of charged particles increases, the number of electrons entering the wire end increases per unit time, and current formed in the interwire arc increases.

Figure 10 shows the change trend diagram of the probe centering time with respect to the plasma arc current.

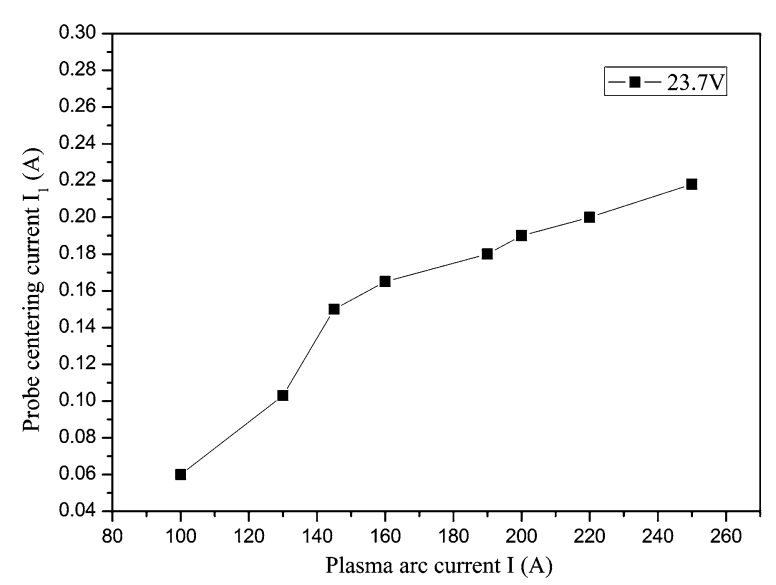

Figure 9 Change trend of the circuit current with respect to the plasma arc current

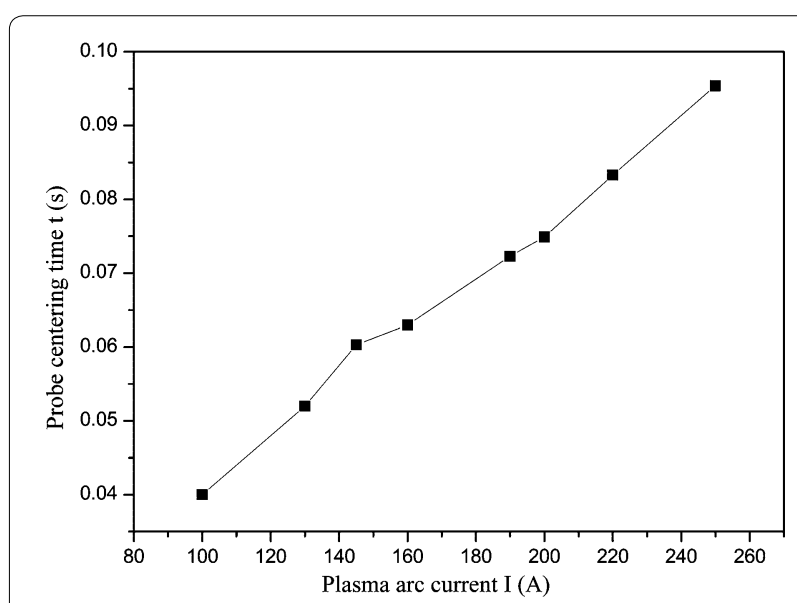

Figure 10 Change trend of the probe centering time with respect to the plasma arc current 
The curve was drawn from Experiment \#2. Using the same probe speed, the influence of the variation of the plasma arc current on the probe centering time was analyzed, and the welding current ranged from 100 to $260 \mathrm{~A}$. If the welding current increases further, the temperature of the plasma arc column increases, and the insulating layer on the surface of the probe is burned, affecting the results of the experimental measurement. As shown in the diagram, as the welding current increases, the probe centering time increases.

The plasma arc column is the main channel of the charged particles. With the increase in the welding current, on one hand, the number of charged particles increases, and the channel becomes wide. On the other hand, the high-speed motion of the charged particles produces the transverse collision component, which also broadens the channel. The plasma arc column extends along the arc center; thus, the probes touches the edge of the arc in advance, and the probe centering time increases correspondingly. In the cross-coupling arc welding, increasing the plasma arc current mainly results in an increase in the width of the main arc column and causes the two wires to enter the plasma arc in advance. Under the condition that the burning point of the wires remains constant, the residence time of the wire in the plasma arc increases, which helps with the full preheating of the wires by the plasma arc.

According to the results of Experiment \#2, as shown in Figures 9 and 10, the probe centering current and time are approximately proportional to the plasma arc welding current when the probe circuit parameters are constant and the plasma welding current is increased. The main difference of the inter-wire arc between the crosscoupling arc and the two-wire indirect arc is that for the cross-coupling arc, the inter-wire arc is formed in the plasma arc, and the effect of the plasma arc must be considered. If the inter-wire arc is fed with a constant voltage power supply and constant wire feeding speed, when the inter-wire arc welding parameters are constant and the plasma arc welding current increases, the heat generation inside the plasma arc increases, and the resistance of the inter-wire arc decreases. At this time, the interwire arc voltage remains unchanged, and the arc current increases. With the increase in the plasma arc power and the inter-wire arc power, the heat production in the cathode and anode regions of the inter-wire arc increases, the melting speed of the welding wire increases, and the stable droplet transition point at the end of the wire deviates from the center line of the plasma arc to maintain the stability of the inter-wire arc. The stable droplet transition point of the inter-wire arc wire in the plasma arc is relatively fixed and is mainly affected by the variation of the welding parameters of the inter-wire arc and the spatial position of the wire in the plasma arc. The arcing time is set as the time from the initial contact at the edge of the plasma arc to the stable droplet transition point. With the increase in the plasma arc current, the area of the arc cross-section increases, which means that the wire contacts the edge of the plasma arc earlier. The stable droplet transition point of the wire basically remains unchanged, resulting in a longer arcing time.

According to the experimental results in Figures 7, 9, and 10, when the plasma arc background is constant, the inter-wire arc changes the welding current by adjusting its wire feeding speed or input voltage, causing the heat production power of the wire end to increase or decrease. The stable droplet transition point of the wire deviates from or moves closer to the center line of the plasma arc, balancing the heat generation between the cathode and anode of the inter-wire arc with the heat provided to the wire by the plasma arc. When the parameters of the inter-wire arc welding remain unchanged, the effect of the plasma arc on the inter-wire arc is embodied in two aspects. On one hand, the increase in the plasma welding current increases the inter-wire arc current and causes the stable droplet transition point of the inter-wire arc to deviate from the plasma arc center line. That is, the interval between the wires increases. When the inter-wire arc is used with an alternating current, the plasma arc current is increased to increase the inter-wire arc re-arcing time.

\subsection{Influence of Space Position of Inter-wire Arc on Inter-wire Arc}

Figure 11 shows the change trend of the probe centering current with respect to the distance between the nozzle and the workpiece. The curve was drawn from

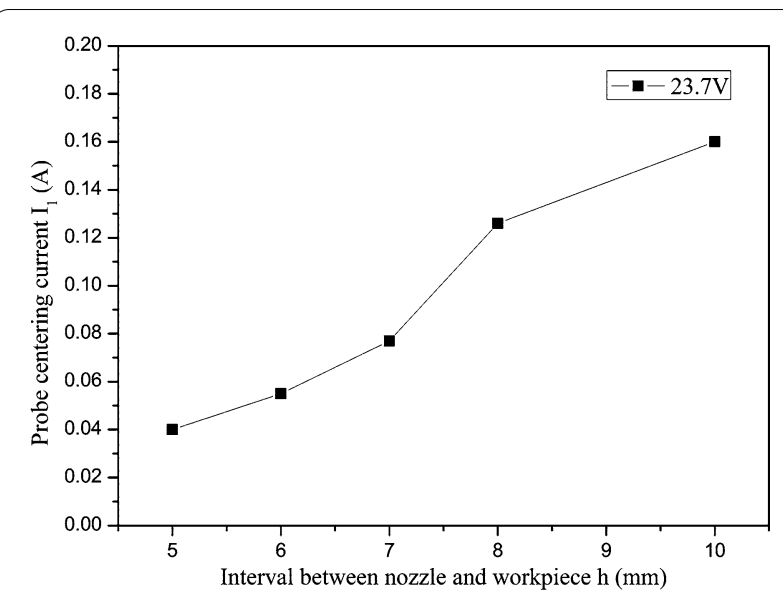

Figure 11 Change trend of the probe centering current with respect to the interval variation between the nozzle and the workpiece 
Experiment \#3, and only the influence of the distance on the centering current in the probe is considered. As shown in Figure 11, the interval significantly affects the measurement results. With the increase in the interval, the probe centering current increases.

A larger interval between the nozzle and the workpiece yields a longer plasma arc and higher arc voltage. Larger amounts of heat generated by the arc column and absorbed by the electrons at the probe tip make it easier for electrons to escape from the probe surface. Moreover, the gas ionization degree increases, and the number of charged particles increases accordingly. A larger amount of electrons entering the probe tip per unit time results in a larger probe-centering current. For different intervals, the variation of the probe centering current is different. When the interval is greater than $8 \mathrm{~mm}$, the current does not change significantly with the increase in the inter$\mathrm{val}$, as in the case of the previous interval of 5-7 $\mathrm{mm}$; the change becomes very gentle. When the interval increases, the heat-dissipation area of the plasma arc column expands, and the heat-dissipation power of the arc column increases. Additionally, the energy amplitude of the gas ionization and the absorption heat of the probe tip become limited, and the probe centering current increases slightly. When the cross-coupling arc welding method is used, the inter-wire arc is controlled by the constant voltage characteristic, and the plasma arc is controlled by the constant current characteristic. At a certain wire feed rate, when the plasma arc length increases, the thermal power of the arc column increases. Additionally, the number of charged particles in the coupling arc volume increases, and the inter-wire arc current increases. The increase in the plasma arc length is accompanied by the expansion of the arc column dissipation area; thus, the increase in the inter-wire current becomes gradual.

\section{Conclusions}

This paper reports a method employing probes rotating across a plasma arc to indirectly study the inter-wire arc in a cross-coupling arc using the probe circuit. Taking the probe centering current and the input voltage as physical quantities, the electrical characteristics of the inter-wire arc were analyzed.

In the experiment, the probe centering current increased with the increase in the input voltage when the plasma arc background was fixed and the probe speed was fixed. At the same input voltage, a higher probe speed yielded a smaller probe current. When the parameters of the probe loop remained unchanged, the probe centering current and time were approximately proportional to the plasma arc current. When the distance between the nozzle and the workpiece was increased, the probe centering current increased, but the arc length also increased. Obviously, the slope of the current rise decreased.

In the cross-coupling arc welding process, if the interwire arc employs a constant voltage source and constant speed wire feed, the inter-wire arc current can be changed by changing the welding parameters of the interwire arc or the plasma arc current. This allows adjustment of the thermal power of the cathode and anode of the inter-wire arc and makes the stable droplet transition point deviate from or move closer to the center line of the plasma arc. In this way, a balance between the heat produced by the inter-wire arc and the heat provided by the plasma arc can be realized.

\section{Authors' Contributions}

ZL applied in model and tested program design, SD did experiments and wrote the manuscript, FJ provided ideas and was in charge of the whole experiments, CL assisted with sampling and laboratory analyses. All authors read and approved the final manuscript.

\section{Acknowledgements}

The authors sincerely thanks to Professor Shujun Chen of Beijing University of Technology for his critical discussion and reading during manuscript preparation

\section{Authors' Information}

Zhenyang Lu, born in 1957, is currently a professor at Beijing University of Technology, China. He received his PhD degree from Beijing University of Technology, China, in 2006. His research interests include welding of high strength steel, welding of light alloy, and arc welding power source and control.

Shanwen Dong, born in 1991, is currently a PhD candidate at Beijing University of Technology, China. He received his master degree from Jiangsu University of Science and Technology, China, in 2013. His research interests include welding arc physics and welding power source.

Fan Jiang, born in 1987, is currently an associate professor at Beijing University of Technology, China. He received his PhD degree from Beijing University of Technology, China, in 2014. His research interests include welding arc physics, innovative welding technology and advanced welding method and application.

Cheng Li, born in 1994, is currently a master candidate at Beijing University of Technology, China.

\section{Competing Interests}

The authors declare that they have no competing interests.

\section{Funding}

Supported by National Natural Science Foundation of China (Grant No. 51875004), Beijing Municipal Natural Science Foundation of China (Grant No. 3172004) and State Key Lab of Advanced Welding and Joining, Harbin Institute of Technology (Grant No. AWJ-16-M06).

Received: 7 March 2018 Accepted: 4 March 2019

Published online: 13 March 2019

\section{References}

[1] J Ogbemhe, K Mpofu. Towards achieving a fully intelligent robotic arc welding: a review. Industrial Robot An International Journal, 2015, 42(5): 475-484.

[2] B Q Cong, Y Su, B J Qi, et al. Development of pulsed arc welding technology for aluminum alloy. Aeronautical Manufacturing Technology, 2016, 59(11): 41-46. (in Chinese) 
[3] DW Deng, R Chen, H C Zhang. Present situation and development trend of plasma surfacing technology. Journal of Mechanical Engineering, 2013, 49(7): 106-112. (in Chinese)

[4] J C Yan, C L Yang, H J Liu, et al. Research status and scientific problems of ultrasonic composite welding. Journal of Mechanical Engineering, 2015, 51(24): 41-49. (in Chinese)

[5] X M Cui, L H Li, Y H Zhang. Research status of high efficiency welding technology. New Technology \& New Process, 2004(7): 32-34.

[6] K Li, Y M Zhang. Consumable double-electrode GMAW. Part II: monitoring, modeling, and control. Welding Journal, 2008, 87(2): 44-50.

[7] K Li, Y M Zhang. Consumable double-electrode GMAW. Part I: the process. Welding Journal, 2008, 87(1): 11-17.

[8] G H Ma, Y M Zhang. A way to weld sheet metal with double-electrode GMAW. Advanced Materials Research, 2013, 651: 333-337.

[9] M A Chen, C S Wu, YM Zhang, et al. Analysis of active control of metal transfer in modified pulsed GMAW. Science \& Technology of Welding \& Joining, 2013, 12(1): 10-14.

[10] J S Chen, Y Lu, X R Li, et al. Gas tungsten arc welding using an arcing wire. Welding Journal, 2012, 91(10): 261-269.

[11] RY Zhang, F Jiang, S J Chen, et al. Influence of bypass power mode on electrical properties and droplet transition of arcing-wire PAW. Transactions of the China Welding Institution, 2017, 38(2): 41-46. (in Chinese)

[12] S J Chen, S L Zhang, N Huang, et al. Droplet transfer of arcing-wire pulse GTAW. Transactions of the China Welding Institution, 2017, 38(1): 17-21. (in Chinese)

[13] S J Chen, S L Zhang, N Huang, et al. Droplet transfer in arcing-wire GTAW. Journal of Manufacturing Processes, 2016, 23:149-156.

[14] S J Chen, G Q Men, Y X Song, et al. Bypass coupling arc (Arcing-wire PAW) high-speed welding process. Transactions of the China Welding Institution, 2017, 38(6): 1-5. (in Chinese)

[15] S J Chen, L Zhang, X P Wang, et al. Stability of cross arc process-a preliminary study. Weld Journal, 2015, 94(5): 158-168.

[16] S J Chen, L Zhang, X P Wang, et al. Feasibility study of cross arc welding process. AWS Professional Program and Poster Session, Chicago 2013.

[17] S J Chen, L Zhang, N Huang, et al. Gas tungsten arc welding with cross AC arcing twin wires. US patent application no. US 9457420 B2, 2016

[18] RY Zhang, F Jiang, S J Chen. Comparison of energy acted on workpiece among twin-body plasma arc welding, non-transferred plasma arc welding and plasma arc welding. Journal of Manufacturing Process, 2016, 24: 152-160.
[19] S J Chen, F Jiang, Y S Lu, et al. Separation of arc plasma and current in electrical arc. Weld Journal, 2013, 93(7): 253-261.

[20] S J Chen, F Jiang, J L Zhang, et al. Measurement and analysis of plasma arc components. Journal of Manufacturing Science \& Engineering, 2015, 137(1): 011006-1-9.

[21] J Wang, K Kusumoto, K Nezu. Analysis of electrical characteristics for hybrid pulsed micro-tungsten inert gas welding arc. Science \& Technology of Welding \& Joining, 2013, 9(4): 369-373.

[22] Vikas Kumar, N Chandrasekhar, SK Albert, et al. Study of manual metal arc welding using digital storage oscilloscope. National Weld Meeting, 2014.

[23] S S Hu, H Zhang, Z J Wang, et al. The arc characteristics of cold metal transfer welding with AZ31 magnesium alloy wire. Journal of Manufacturing Processes, 2016, 24: 298-306.

[24] F Jiang, Y F Li, S J Chen. Current situation and prospects of welding arc monitoring technology. Journal of Mechanical Engineering, 2018, 54(2): 16-26. (in Chinese)

[25] Y B Li, T Lu, L Zhu, et al. Electrostatic probe analysis of sheet like tungsten inert arc current carrying zone. Transactions of the China Welding Institution, 2015, 36 (12): 22-26. (in Chinese)

[26] Y B Li, L Zhu, XX Jiang. Diagnosis and analysis of TIG arc current zone with low disturbance electrostatic probe. Journal of Lanzhou University of Technology, 2014(1): 24-28. (in Chinese)

[27] Y B Li, X Li, Q F Shi, et al. Differential analysis of low disturbance electrostatic probe for the temperature of TIG arc current zone. Transactions of the China Welding Institution, 2017, 38(5): 26-30. (in Chinese)

[28] S J Chen, R Y Zhang, F Jiang, et al. A progressive device and method for detecting the arc characteristics. Chinese Patent: CN106238870A, 2016-12-21.

[29] F Jiang, R Y Zhang, S J Chen, et al. A sweeping device and method for detecting the arc characteristic. Chinese Patent: CN106199278A, 2016-12-07.

[30] L Lu. Behavior research on high frequency double-pulse TIG welding arc. Shenyang: Shenyang University of Technology, 2014.

[31] Y Chang, M Liu, L Lu, et al. The influence of longitudinal magnetic field on the CO2 arc shape. Plasma Science and Technology, 2015, 17(4): 321-326.

[32] J B Wang, X Q LV, Y Wang. Hybrid arc model of pulsed TIG welding. Transactions of the China Welding Institution, 2015, 36(9): 26-30.

[33] T S Dong, L J Yang, J J Liang. MATLAB simulation of dynamic characteristics of small current pulsed TIG welding arc. Welding Technology, 2003(3): 11-13. (in Chinese)

\section{Submit your manuscript to a SpringerOpen ${ }^{\odot}$ journal and benefit from:}

- Convenient online submission

- Rigorous peer review

- Open access: articles freely available online

- High visibility within the field

- Retaining the copyright to your article

Submit your next manuscript at $\boldsymbol{\nabla}$ springeropen.com 